\title{
中医康复从业人员科研水平的 “知信行”现状及对策研究
}

\author{
宋 健 ${ }^{1,2}$, 刘志臻 $1^{*}$, 吴劲松 ${ }^{1,2,3}$, 彭 娟 ${ }^{1}$, 陈 斌 ${ }^{4}$, 黄 佳 ${ }^{1,2,3}$ \\ 1 福建中医药大学, 福建 福州 350122; \\ 2 福建省康复技术协同创新中心, 福建 福州 350122 ; \\ 3 国家中医药管理局中医康复研究中心, 福建 福州 350122; \\ 4 福建中医药大学附属人民医院, 福建福州 350004 \\ *通信作者:刘志䠈, E-mail : lzz@fjtcm.edu.cn
}

收稿日期: 2020-01-03; 接受日期 : 2020-02-25

基金项目: 国家中医药管理局科研管理专项课题(GZY-KJS-2017-07)

DOI : 10.3724/SP.J.1329.2020.02013

开放科学 (资源服务) 标识码 $($ OSID):

摘要 目的: 分析不同级别康复医疗机构科研人员 “知识一信念一行动”现状和存在问题, 并提出相应的对策。 方法: 选择 34 个省、市、自治区的中医康复从业人员作为调查对象, 通过自愿回应样本的方式进行《中医康 复从业人员科研水平知信行调查问卷》调查, 样本按医院级别分为三级医院组和非三级医院组, 比较 2 组中 医康复从业人员科研知信行现状。结果: 共回收有效问卷 494 份, 问卷有效率 $81.79 \%$, 其中三级医院组 369 例, 非三级医院组 125 例。在知识维度中, 三级医院组在常用文献数据库检索、基于康复知识进行科研设计 的能力高于非三级医院组 $(P<0.05)$, 而在康复专业相关文献阅读、科研统计学分析方面无明显差异 $(P>$ $0.05)$; 在信念维度中, 三级医院科研过程中遇到的困难明显低于非三级医院组 $(P<0.05)$, 而在科研的重要 性认识、从事科研的原因、遇到困难时寻找相关知识等方面无明显差异 $(P>0.05)$; 在行动维度中, 三级医院 组科研投入时间、参与高级别研究棵题机会、国内期刊论文产出数量明显高于非三级医院组 $(P<0.05)$, 而在 科研方案调整、论文规范写作、承担高级别课题机会、SCI 源期刊论文发表数量、参与科研相关培训次数等方 面无明显差异 $(P>0.05)$; 此外, 典型相关分析结果显示医院分级、知识维度和信念维度与行动维度呈正相关 关系, 知识和信念维度的认知程度越高, 对行动维度的影响力也越大。结论: 不同级别康复医疗机构间人才 分配不均匀、科研水平参差不齐。非三级医院的中医康复从业者整体科研能力偏弱, 而三级医院尚未发挥出 相应的科研与人才优势。因此, 有必要加强科研的知识获取、信念支撑和实践应用, 培养高水平的中医康复 临床科研型双向人才, 进而促进中医康复学科的发展。

关键词中医康复; 科研水平; 知信行; 问卷调查

中医康复独特地融合了中医传统康复与现代 康复医学思想, 在康复服务实践中具有切实的临床 疗效和广泛的群众基础, 对全民健康的维持与促进 具有重要作用 ${ }^{[1-3]}$ 。科研是推进中医康复临床发展的 基石和动力, 但目前中医康复从业者的科研能力尚 存短板, 相关临床实践进人高级别循证指南的成果 仍较少 ${ }^{[4]}$, 一定程度上制约了中医康复学科的发展。
“知信行”理论 (knowledge-attitude-practice, KAP) 拓展至中医康复领域, 将科研过程总结为获取知识、 产生信念和主动实践 3 个连续过程, 即 “知识-信 念-行为” [5]。通过康复临床实践发现问题, 借助科研 手段与高新技术探索并解决问题, 继而转化出更好 的科研成果 ${ }^{[6]}$ 。本研究面向全国 34 个省、市、自治区 的康复医疗机构的中医康复从业人员发放问卷进

引用格式: 宋健, 刘志漛, 吴劲松, 等. 中医康复从业人员科研水平的“知信行”现状及对策研究 $[\mathrm{J}]$. 康复学报, 2020,30(2): 149-154.

SONG J,LIU Z Z, WU J S, et al. Research on the status of KAP and countermeasures of the scientific research level of TCM rehabilitation practitioners [J]. Rehabilitation Medicine, 2020,30(2):149-154.

DOI: $10.3724 /$ SP.J.1329.2020.02013 
行调查,分析和比较不同级别的康复医疗机构科研 水平的知信行现状, 以期为制定中医康复从业人员 的科研促进计划提供依据。

\section{1 资料与方法}

\section{1 调查对象}

选择全国 34 个省、市、自治区的康复医疗机构 的中医康复从业人员作为研究对象, 具体包括从事 中医 (传统) 康复的康复医师和康复治疗师。

\section{2 调查问卷设计}

《中医康复从业人员科研水平知信行调查问 卷》是课题组在文献调研和实地调研的基础上根据 中医康复从业人员科研情况设计的, 主要包括基本 资料和科研概况 2 个部分, 其中基本资料包括性别、 年龄、学历、工作单位 (三级医院和非三级医院)、从 业类型、从事中医康复时间、职称等; 科研概况包括 知识、信念、行动等 3 个维度内容。问卷的专家权威 程度系数为 0.9125 , 内部一致性信度克朗巴哈系数 Cronbach's $\alpha=0.866$, 结构效度 $\mathrm{KMO}=0.870, P<$ 0.001 。该问卷内容设计较为科学、信效度良好。

\section{3 问卷调查}

2017 年 9 月通过在线问卷的方式, 采用自愿回 应样本的抽样方法。所有调查对象均在自愿情况下 独立完成问卷, 并由审核人员负责核对问卷的填写 情况, 对填写内容的可靠性做出判断。

\section{4 统计学方法}

首先, 应用 Excel 2016 对问卷数据进行整理与 清洗并建立数据库。其次, 应用 SPSS 24.0 软件进行 数据分析。计数资料采用 $\chi^{2}$ 检验, 变量间的相关性 采用典型相关分析方法。 $P<0.05$ 为差异有统计学 意义。

\section{2 结 果}

\section{1 一般资料}

共收回问卷 604 份, 其中有效问卷 494 份, 有 效率是 $81.79 \%$ 。华中、华北、华东、华南、西南、西北、 东北地区分别回收 $73 、 18 、 217 、 49 、 35 、 56 、 46$ 份问 卷。回收的问卷按医院级别分为三级医院组和非三 级医院组,每组分别 369 份、125 份。2 组一般资料 比较情况，见表 1 。

表 12 组一般资料比较

Table 1 Comparison of general data between two groups

\begin{tabular}{|c|c|c|c|c|c|c|c|c|c|c|c|}
\hline 项 目 & 选 项 & $\begin{array}{c}\text { 非三级医院 } \\
(n=125)\end{array}$ & $\begin{array}{l}\text { 三级医院 } \\
(n=369)\end{array}$ & $\chi^{2}$ 值 & $P$ 值 & 项 目 & 选 项 & $\begin{array}{c}\text { 非三级医院 } \\
(n=125)\end{array}$ & $\begin{array}{l}\text { 三级医院 } \\
(n=369)\end{array}$ & $\chi^{2}$ 值 & $P$ 值 \\
\hline \multirow{2}{*}{ 性别 } & 男 & 75 & 181 & \multirow{2}{*}{4.48} & \multirow{2}{*}{$0.038^{1)}$} & \multirow{2}{*}{ 从业类型 } & 中医康复医师 & 80 & 200 & \multirow{2}{*}{3.65} & \multirow{2}{*}{0.061} \\
\hline & 女 & 50 & 188 & & & & 治疗师 & 45 & 169 & & \\
\hline \multirow{4}{*}{ 年龄/岁 } & $\geqslant 50$ & 6 & 21 & \multirow{4}{*}{17.33} & \multirow{4}{*}{$0.001^{1\rangle}$} & \multirow{4}{*}{ 学历 } & 博士 & 0 & 28 & \multirow{4}{*}{79.75} & \multirow{4}{*}{$0.001^{17}$} \\
\hline & $40 \sim 49$ & 35 & 48 & & & & 硕士 & 16 & 140 & & \\
\hline & $30 \sim 39$ & 38 & 107 & & & & 本科 & 68 & 178 & & \\
\hline & $<30$ & 46 & 193 & & & & 专科 & 41 & 23 & & \\
\hline \multirow{5}{*}{$\begin{array}{l}\text { 从事中 } \\
\text { 医康复 } \\
\text { 时间/年 }\end{array}$} & $>20$ & 12 & 27 & \multirow{5}{*}{8.80} & \multirow{5}{*}{0.066} & \multirow{5}{*}{ 职称 } & 高级 & 18 & 62 & \multirow{5}{*}{6.66} & \multirow{5}{*}{0.083} \\
\hline & $16 \sim 20$ & 15 & 21 & & & & 中级 & 33 & 79 & & \\
\hline & $11 \sim 15$ & 15 & 33 & & & & 初级 & 53 & 191 & & \\
\hline & $6 \sim 10$ & 25 & 75 & & & & 其他 & 21 & 37 & & \\
\hline & $\leqslant 5$ & 58 & 213 & & & & & & & & \\
\hline
\end{tabular}

注: 与非三级医院比较, 1) $P<0.05$ 。

Note: Compared with non tertiary hospitals, 1) $P<0.05$.

\section{2 中医康复从业人员科研“知信行”现状}

\subsection{1 科研知识维度情况 见表 2 。}

2.2.2 科研信念维度情况 见表 3 。

2.2.3 科研行动维度情况 见表 4 。

2.2.4 医院分级、知识和态度对科研行为的影响 第 1 组典型相关系数 0.581 , 显著性 $(P=0.001<0.05)$, 解释能力达 0.338 ; 第 2 组典型相关系数 0.085 , 显
著性 $(P=0.310>0.05)$; 第 3 组典型相关系数 0.051 , 显著性 $(P=0.261>0.05)$ 。第 1 组典型相关变量的 自我解释能力最高, 为 $100 \%$ 。医院分级、文献检索 和制定科研计划可通过第 1 组典型变量显著影响 到行为, 其对行为可以相互解释的总变异量为 $34.8 \%$, 行动通过 3 个条目可以解释医院分级、文献检索和 制定科研计划总变异量的 $17.6 \%$ 。见图 1。 
表 2 中医康复从业人员科研知识维度情况

Table 2 Scientific research knowledge dimensions of rehabilitation practitioners of TCM

\begin{tabular}{|c|c|c|c|c|c|c|}
\hline 组 别 & $n$ & 选项 & $\begin{array}{l}\text { 使用常见文献数 } \\
\text { 据库检索资料 }\end{array}$ & $\begin{array}{l}\text { 读懂康复专 } \\
\text { 业相关文献 }\end{array}$ & $\begin{array}{l}\text { 在康复知识理论 } \\
\text { 基础上科研设计 }\end{array}$ & $\begin{array}{l}\text { 选择合适统计方 } \\
\text { 法分析科研数据 }\end{array}$ \\
\hline \multirow{5}{*}{ 非三级医院组 } & \multirow{5}{*}{125} & 完全能做到 & 7 & 7 & 2 & 5 \\
\hline & & 经常做到 & 11 & 9 & 18 & 10 \\
\hline & & 尚可做到 & 26 & 29 & 28 & 39 \\
\hline & & 较少做到 & 44 & 46 & 46 & 41 \\
\hline & & 无法做到 & 37 & 34 & 31 & 30 \\
\hline \multirow{5}{*}{ 三级医院组 } & \multirow{5}{*}{369} & 完全能做到 & 20 & 19 & 18 & 17 \\
\hline & & 经常做到 & 58 & 48 & 45 & 46 \\
\hline & & 尚可做到 & 118 & 116 & 131 & 120 \\
\hline & & 较少做到 & 119 & 112 & 112 & 109 \\
\hline & & 无法做到 & 54 & 74 & 63 & 77 \\
\hline$\chi^{2}$ 值 & & & 18.806 & 8.318 & 11.957 & 2.521 \\
\hline$P$ 值 & & & $0.001^{1)}$ & 0.081 & $0.018^{1)}$ & 0.641 \\
\hline
\end{tabular}

注: 与非三级医院比较, 1) $P<0.05$ 。

Note: Compared with non tertiary hospitals, 1) $P<0.05$.

表 3 中医康复从业人员科研信念维度情况

Table 3 Scientific research attitude dimensions of rehabilitation practitioners of TCM

\begin{tabular}{|c|c|c|c|c|c|c|c|c|c|c|c|}
\hline 项 目 & 选 项 & $\begin{array}{c}\text { 非三级医院 } \\
(n=125)\end{array}$ & $\begin{array}{c}\text { 三级医院 } \\
(n=369)\end{array}$ & $\chi^{2}$ 值 & $P$ 值 & 项 目 & 选 项 & $\begin{array}{c}\text { 非三级医院 } \\
(n=125)\end{array}$ & $\begin{array}{l}\text { 三级医院 } \\
(n=369)\end{array}$ & $\chi^{2}$ 值 & $P$ 值 \\
\hline 科研重 & 非常重要 & 63 & 210 & \multirow{5}{*}{4.191} & \multirow{5}{*}{0.123} & \multirow{5}{*}{$\begin{array}{l}\text { 进行科研 } \\
\text { 主要原因 }\end{array}$} & 总结临床经验 & 99 & 263 & \multirow{5}{*}{8.608} & \multirow{5}{*}{0.072} \\
\hline 要性认 & 重要 & 43 & 126 & & & & 晋升职称 & 68 & 260 & & \\
\hline \multirow[t]{4}{*}{ 识 } & \multirow[t]{3}{*}{ 一般 } & \multirow[t]{3}{*}{19} & \multirow[t]{3}{*}{33} & & & & 服务康复事业 & 76 & 204 & & \\
\hline & & & & & & & 单位要求 & 38 & 153 & & \\
\hline & & & & & & & 报道科研成果 & 35 & 136 & & \\
\hline & 缺乏科研技巧 & 85 & 284 & \multirow{5}{*}{16.64} & \multirow{5}{*}{$0.002^{1)}$} & \multirow{5}{*}{$\begin{array}{l}\text { 遇到难题 } \\
\text { 寻找相关 } \\
\text { 知识 }\end{array}$} & 完全能做到 & 21 & 42 & \multirow{5}{*}{8.251} & \multirow{5}{*}{0.083} \\
\hline 科研遇 & 缺乏科研知识 & 88 & 273 & & & & 经常做到 & 43 & 125 & & \\
\hline 到主要 & 缺之科研资金 & 90 & 230 & & & & 尚可做到 & 37 & 154 & & \\
\hline 困难 & 获取信息困难 & 81 & 203 & & & & 较少做到 & 21 & 43 & & \\
\hline & 缺乏领导支持 & 60 & 92 & & & & 无法做到 & 3 & 5 & & \\
\hline
\end{tabular}

注: 与非三级医院比较, 1) $P<0.05$ 。

Note: Compared with non tertiary hospitals, 1) $P<0.05$.

\section{3 讨 论}

\section{1 中医康复从业人员科研发展机遇}

本研究调查的中医康复从业人员中, 从午龄上 看, 40 岁及以下占 $77.73 \%$, 说明青年中医从业者是 中医康复事业的主力军; 从学历结构上看, 本科及 以上学历占 $87.04 \%$, 其中硕、博士占 $37.25 \%$, 与既 往调查比较, 高学历从业人员数量呈现明显增长 趋势 ${ }^{[7-8]}$; 从职称结构和工作年限上看, 中级和初级 职称从业人员占 $72.06 \%$, 从业时间 $<10$ 年占 $75.10 \%$,
这提示高学历的青年中医从业人员是当前各级别 康复医疗机构的中坚力量, 他们大多职称还不高, 却对科研工作重要性的认识普遍较高, 目前中医康 复从业人员的科研工作存在巨大发展机遇与挑战, 如果能引导康复从业人员在具备扎实的中医辩证 思维和临床治疗能力基础上进一步提升科研素质 与能力, 在未来 10 年他们将有可能成为各级别康复 医疗机构的中流砥柱 ${ }^{[9-10]}$ 。

\section{2 中医康复从业人员科研水平提升措施}

3.2.1 因势利导、因才施策, 提升科研能力本研 
表 4 中医康复从业者科研行动维度情况

Table 4 Scientific research practice dimensions of rehabilitation practitioners of TCM

\begin{tabular}{|c|c|c|c|c|c|c|c|c|c|c|c|}
\hline 项 目 & 选 项 & $\begin{array}{c}\text { 非三级医院 } \\
(n=125)\end{array}$ & $\begin{array}{l}\text { 三级医院 } \\
(n=369)\end{array}$ & $\chi^{2}$ 值 & $P$ 值 & 项 目 & 选 项 & $\begin{array}{c}\text { 非三级医院 } \\
(n=125)\end{array}$ & $\begin{array}{l}\text { 三级医院 } \\
(n=369)\end{array}$ & $\chi^{2}$ 值 & $P$ 值 \\
\hline \multirow{5}{*}{$\begin{array}{l}\text { 实践与设 } \\
\text { 计不符, } \\
\text { 能调整研 } \\
\text { 究方案 }\end{array}$} & 完全做到 & 6 & 16 & \multirow{5}{*}{6.652} & \multirow{5}{*}{0.155} & \multirow{5}{*}{$\begin{array}{l}\text { 能够规范 } \\
\text { 书写论文 }\end{array}$} & 完全做到 & 9 & 21 & \multirow{5}{*}{8.331} & \multirow{5}{*}{0.08} \\
\hline & 经常做到 & 16 & 56 & & & & 经常做到 & 15 & 54 & & \\
\hline & 尚可做到 & 35 & 141 & & & & 尚可做到 & 36 & 151 & & \\
\hline & 较少做到 & 36 & 90 & & & & 较少做到 & 42 & 92 & & \\
\hline & 无法做到 & 32 & 66 & & & & 无法做到 & 23 & 51 & & \\
\hline & 国家级 & 2 & 22 & \multirow{5}{*}{9.37} & \multirow{5}{*}{0.052} & \multirow{5}{*}{$\begin{array}{l}\text { 参与最高 } \\
\text { 研究课题 } \\
\text { 级别 }\end{array}$} & 国家级 & 10 & 83 & \multirow{5}{*}{22.32} & \multirow{5}{*}{$0.001^{1)}$} \\
\hline 承担最高 & 省部级 & 11 & 32 & & & & 省部级 & 10 & 48 & & \\
\hline 研究课题 & 厅局级 & 6 & 30 & & & & 厅局级 & 7 & 30 & & \\
\hline \multirow[t]{2}{*}{ 级别 } & 院所级 & 12 & 55 & & & & 院所级 & 14 & 42 & & \\
\hline & 无 & 94 & 230 & & & & 无 & 84 & 166 & & \\
\hline 以 第 一 & $\geqslant 10$ & 0 & 2 & \multirow{5}{*}{1.156} & \multirow{5}{*}{0.885} & \multirow{5}{*}{$\begin{array}{l}\text { 以第一 } \\
\text { (通信) 作 } \\
\text { 者发表国 } \\
\text { 内论文数 }\end{array}$} & $\geqslant 10$ & 4 & 27 & \multirow{5}{*}{19.83} & \multirow{5}{*}{$0.001^{1)}$} \\
\hline (通信) 作 & $7 \sim 9$ & 1 & 2 & & & & $7 \sim 9$ & 5 & 12 & & \\
\hline 者发表 & $4 \sim 6$ & 1 & 5 & & & & $4 \sim 6$ & 5 & 37 & & \\
\hline SCI 论文 & $1 \sim 3$ & 13 & 34 & & & & $1 \sim 3$ & 27 & 125 & & \\
\hline 数 & 0 & 110 & 326 & & & & 0 & 84 & 168 & & \\
\hline & $>60$ & 2 & 13 & \multirow{5}{*}{14.09} & \multirow{5}{*}{$0.003^{1)}$} & \multirow{5}{*}{$\begin{array}{l}\text { 参加科研 } \\
\text { 培训次数 }\end{array}$} & $>3$ 次/年 & 7 & 28 & \multirow{5}{*}{5.75} & \multirow{5}{*}{0.218} \\
\hline 每年从事 & $31 \sim 60$ & 5 & 39 & & & & $2 \sim 3$ 次/年 & 12 & 38 & & \\
\hline 科研时间 & $6 \sim 30$ & 27 & 116 & & & & 1 次/年 & 27 & 105 & & \\
\hline \multirow{2}{*}{ /(d/年) } & $7 \sim 15$ & 91 & 201 & & & & 1 次 $/ 1 \sim 5$ 年 & 28 & 88 & & \\
\hline & & & & & & & < 1 次/5 年 & 51 & 110 & & \\
\hline
\end{tabular}

注: 与非三级医院比较, 1) $P<0.05$ 。

Note: Compared with non tertiary hospitals, 1) $P<0.05$.

\begin{tabular}{l} 
第一组变量自我解释能力 $=100 \%$ \\
重叠量 $=17.6 \%$ \\
\hline
\end{tabular}

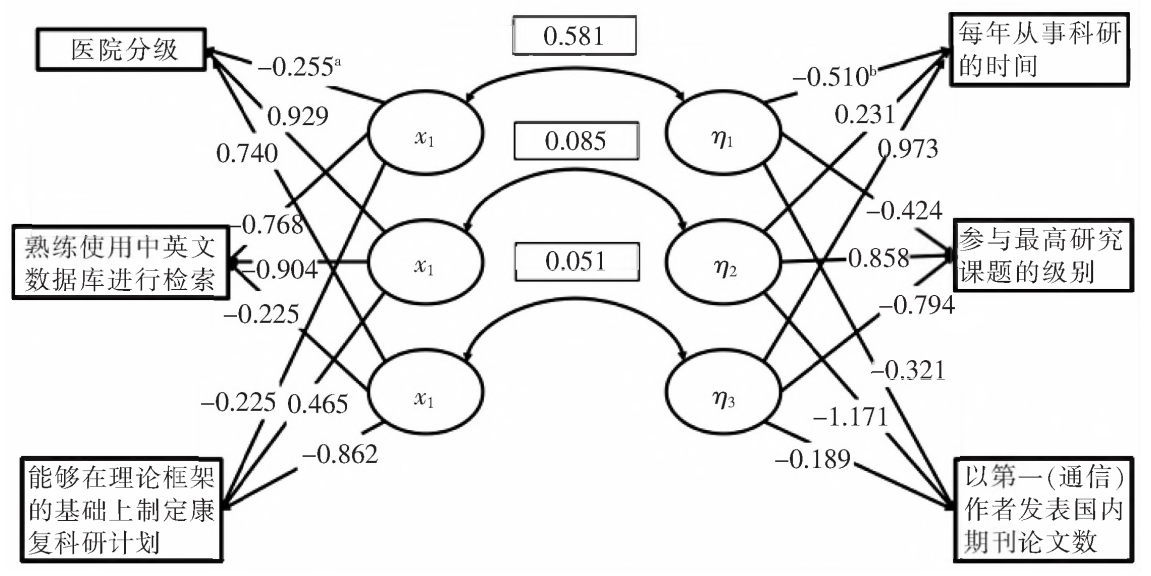

注: $a$ 为第 1 组变量的标准化典型加权系数; $b$ 为第 2 组变量的标准化典型加权系数。

Note: a is the standardized typical weighting coefficient of variable in group 1 ; b is the standardized typical weighting coefficient of variable in group 2 .

图 1 典型相关分析图

Figure 1 Chart of canonical correlation analysis 
究结果显示, $32.59 \%$ 调查对象表示很少有机会参加 科研培训 $(<1$ 次 $/ 5$ 年 $)$ 。这进一步凸显了中医康复 从业人员普遍存在文献检索能力、科研设计能力较 差等问题。文献检索和科研设计是科研思维形成的 重要基础, 文献检索可以帮助科研人员迅速了解学 术界的研究发展动向,而科研设计科学与否决定着 整个科研计划实施的可行性 ${ }^{[11]}$ 。从科研动机看, 大 多数中医康复从业者表示进行科研主要是为了总 结临床经验、晋升职称和为康复事业做贡献。因此, 要提升中医康复从业人员的科研水平应因势利导、 因才施策, 对非三级医院的康复从业人员应重点加 强文献检索、科研设计与技能提升等方面的培训, 引 导他们立足临床康复需求, 重点总结临床经验; 对 三级医院康复从业人员应协调优质资源, 加强合作 共享, 鼓励他们与本单位或业内优秀的康复科研团 队协作,围绕康复治疗难点、学科前沿热点和“高精 尖”康复技术等方面进行研究,力争共同解决 “卡脖 子”问题,逐步完善中医康复医学临床科研标准体 系 ${ }^{[12]}$,促进中医康复学科的发展与建设。

3.2.2 发现问题、突破困境,艻实科研基础科研 信念是科研实施的内在动力 ${ }^{[13-14]}$ 。本研究显示, $89.47 \%$ 受调查对象均认识到科研的重要性, 但他们 往往因为缺乏科研知识、科研技巧、科研资金和获 取信息困难等无法开展科研工作或科研工作进度 缓慢。作为医院科研管理部门应加强调研, 及时发 现本单位中医康复从业者在科研工作中存在的主 要问题, 科学分析问题产生的原因及对策, 为开展 科学研究提供坚实的基础和制度保障。三级医院应 系统性明确科研任务, 合理开展、推进科研相关讲 座或培训,鼓励从业者参加高水平的学术峰会或者 研习班, 提高科研能力和技巧; 同时鼓励骨干科室 组建科研团队, 积极协助提供基础研究或前期立项 资金支持,进一步完善科研平台的建设,为科研提 供制度保障。非三级医院在资金、技术、人才相对薄 弱的情况下,应多措并举努力提升科研人员素质, 如可通过与三级医院、高校等共同做好协同创新与 科研转化工作, 在协作过程中努力培养科研人才; 挖掘基层医院自身优势, 为开展特殊病种、较大规 模临床随机对照研究提供科研资料, 逐步培养本单 位从业人员形成 “见微知著”的科研意识; 同时加强 医院临床诊疗数据信息化、共享化, 以提升科研产 出和科研管理效率 ${ }^{[15]}$

3.2.3 优势互补、资源整合, 促进成果转化 本研 究结果显示, 仅 $11.94 \%$ 的中医康复从业人员表明有
充足的时间从事科研 $(>30 \mathrm{~d} /$ 年)。三级医院的康复 从业人员从事科研的时间更多, 也更有机会参与省 部级以上等高级别科研课题的研究, 国内期刊论文 产出也更高。进一步的典型相关分析结果显示, 医 院级别、数据库检索能力、科研设计能力与从事科 研时间、参与高级别课题研究、论文产出数量等呈 正相关关系。因此, 三级医院应借助其优势的医疗 资源, 积极促进与非三级医院的科研沟通与交流, 积 极发挥临床科研前、后端的联动效应, 实现康复资

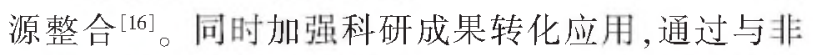
三级医院的合作及时将最新的科研成果应用于临 床, 这不仅能让科研更好地为患者服务, 还有利于 激发康复从业人员的科研主观能动性, 自觉培养科 研意识与科研学习氛围, 从科研基础人手, 寓临床 思维于科研思想之中 ${ }^{[17-18]}$, 通过低成本的科研投入。 与转化, 发展适用于基层的中医康复共性技术及小 型化的中医康复设备 ${ }^{[19]}$, 甚至推动科研创新, 有效 促进中医康复适宜技术和成果在基层推广应用 ${ }^{[20-21]}$ 。

\section{4 小 结}

当前,我国正处于实施“健康中国战略”的关键 时期, 党和国家高度重视发展康复服务, 我国康复

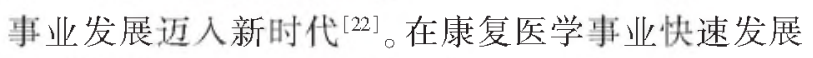
的时代里, 科研水平已经成为康复从业人员能否成 功抓住发展机遇、迎接挑战的关键因素。虽然, 当前 我国各级康复医疗机构康复从业人员科研工作仍 存在许多短板, 但如果能在临床康复诊疗与科学研 究融合上做文章, 补足短板、优势互补、资源共享, 则中医康复科研工作未来可期。

\section{参考文献}

[1]中国老年保健医学研究会老龄健康服务与标准化分会. 中国 高龄脑卒中患者康复治疗技术专家共识 $[\mathrm{J}]$. 中国老年保健医 学, 2019,17(1):3-16.

[2]郑婷婷, 秦萍, 朱路文, 等. 脑卒中康复新策略: 针康法研究进 展 $[J]$. 世界中西医结合杂志, 2018, 13(7): 1034-1036.

[3] 张丹丹, 陶静, 陈立典. 从中医康复发展脉络探讨时代背景对 康复医学发展的影响 [J]. 中医杂志, 2019,60(14):11761180 .

[4]许明, 张泓, 谭洁, 等. 基于现代康复医学理论体系对中医康 复的应用与研究之思考 $[\mathrm{J}]$. 湖南中医药大学学报, 2017,37 (10): 1161-1165.

[5]刘志愫, 许茜, 白乔, 等. 构建基于 KAP 结构的研究生医学统 计学教学实践 $[J]$. 中华医学教育探索杂志, 2017, 16(8):780784 .

[6]赵树华, 郑若楠. “临床是基础, 科研是动力, 人才是核心”: 论 康复发展理念 $[\mathrm{J}]$. 按摩与康复医学, 2016,7(8):75-76. 
[7]任艳苹, 郭琪, 李雨晴, 等. 我国社区康复医疗资源的现状与 需求 $[\mathrm{J}]$. 中国康复医学杂志, 2014,29(8):757-759.

[8] 郑洁皎,俞卓伟, 张炜, 等. 上海市康复医疗资源调查报告 $[\mathrm{J}]$. 中国康复医学杂志, 2013, 28(2):143-147.

[9]王旦, 冯莺, 黄雪燕, 等. 中医康复护士中医护理能力及培训 需求调查 $[J]$. 护理学杂志, 2019,34(6):65-68.

$[10]$ 李夏. 广西康复技术专业人才需求与培养现状调查 $[\mathbf{J}]$. 卫生 职业教育 , 2018,36(16): 137-138.

[11] 穆士卿. 浅论临床医学研究生科研思维的培养 $[\mathrm{J}]$. 中国医刊, 2017,52(1): 107-108.

[12] 章稳, 郭琪, 高连欢, 等. 天津市二、三级医院医疗康复资源 及服务能力调查报告 $[\mathrm{J}]$. 中国康复, 2018, 33(4):330-332.

[13] 白俊敏,白䎦杰, 张铭, 等. 基于课题研究培养康复医学研究 生科研能力的探索 $[\mathrm{J}]$. 中医药导报, 2019,25(9):133-134.

[14] 廖利民. 加强康复医学专业研究生的科研能力培养 $[\mathrm{J}]$. 中国 康复理论与实践, 2016,22(5):618-620.

[15] 起欣欣,李觉,吴恒懪. 社区全科医生科研培养路径的实践与
思考 $[J]$. 中华全科医学, 2019,17(12):1979-1981.

[16] 蒋剑, 张永平, 张永裕, 等. 福建省康复医疗资源现状调查与 发展探讨 $[\mathrm{J}]$. 中国康复医学杂志, 2015,30(4):369-373.

[17] 谭忠貄, 秦彦文. 医学科研院所科技成果转化的问题及对策 $[\mathbf{J}]$. 北京生物医学工程, 2018,37(4):410-412.

[18] 邓生琼, 朱占洲, 张灵, 等.二级医院科研发展困境 $[\mathrm{J}]$. 解放 军医院管理杂志, 2019,26(10): 974-976.

$[19]$ 陈立典. 充分发挥中医在疾病康复中的作用 $[\mathrm{J}]$. 康复学报, $2018,28(2): 1-4$.

[20]郭健, 张雅素, 葛亚博, 等. 提高中医康复学硕士研究生科研 能力的探索 $[\mathrm{J}]$. 中国中医药现代远程教育, 2018, 16(8): $36-38$.

[21] 陈立典. 发展社区康复, 构建低成本广覆盖的健康服务体系 $[\mathrm{J}]$. 康复学报, 2018,28(5):1-4.

[22] 陈立典. 新时代 新视野 新康复 [J]. 康复学报, 2019, 29(4): $1-3$.

\title{
Research on the Status of KAP and Countermeasures of the Scientific Research Level of TCM Rehabilitation Practitioners
}

\author{
SONG Jian ${ }^{1,2}$, LIU Zhizhen ${ }^{1 *}$, WU Jingsong ${ }^{1,2,3}$, PENG Juan ${ }^{1}$, CHEN Bin ${ }^{4}$, HUANG Jia ${ }^{1,2,3}$ \\ ${ }^{1}$ Fujian University of Traditional Chinese Medicine, Fuzhou, Fujian 350122, China; \\ ${ }^{2}$ Fujian Collaborative Innovation Center for Rehabilitation Technology, Fuzhou, Fujian 350122, China; \\ ${ }^{3}$ TCM Rehabilitation Research Center of SATCM, Fuzhou, Fujian 350122, China; \\ ${ }^{4}$ People's Hospital Affiliated of Fujian University of Traditional Chinese Medicine, Fuzhou, Fujian 350004, China \\ *Correspondence: LIU Zhizhen, E-mail: lzz@fitcm.edu.cn
}

\begin{abstract}
Objective: To analyze the status and problems of "knowledge-attitude-practice" (KAP) for rehabilitation practitioners of TCM in different classes of rehabilitation medical institutions, and to propose corresponding countermeasures. Methods: TCM rehabilitation practitioners in 34 provinces, municipalities and autonomous regions were selected as respondents. The questionnaires on the KAP of scientific research level in TCM rehabilitation practitioners were voluntarily responded to the samples. The samples were divided into tertiary hospital group and non-tertiary hospital group according to the hospital level, and compare the status of KAP between two groups. Results: We received a total of 494 valid questionnaires, the efficiency of the questionnaire was $81.79 \%$, with 369 cases in the tertiary hospital group and 125 cases in the non-tertiary hospital group. At the knowledge dimension, compare with non-tertiary hospital group, the ability of database literature retrieval, rehabilitation research and design of the tertiary hospital group were higher $(P<0.05)$, while there were no significant differences in rehabilitation literature reading and statistical analysis of scientific research $(P>0.05)$. At the attitude dimension, the difficulties in the scientific research process of tertiary hospitals were significantly lower than those in non-tertiary hospital groups $(P<0.05)$, and there were no significant differences in importance recognition of the scientific research, reasons for scientific research, solutions when encounter difficulties $(P>0.05)$. At the practice dimension, the time spent in scientific research, the opportunity to participate in high-level research projects, and the number of domestic journal paper output were significantly higher than those in non-tertiary hospital groups $(P<0.05)$, and there were no significant differences in adjustment of scientific research plan, the opportunity to undertake high-level subjects, the number of papers in SCI source journal, and the number of scientific research-related training participations $(P>0.05)$. In addition, the results of canonical correlation analysis showed that practice dimension was positive correlation to hospital classification, knowledge dimension and attitude dimension, the higher the knowledge and attitude dimensions, the greater the influence on the practice dimensions. Conclusion: There were uneven distribution of talents and uneven level of scientific research in different class of rehabilitation medical institutions. The overall scientific research capabilities of TCM rehabilitation practitioners in non-tertiary hospitals is weak, while tertiary hospitals have not been given full play to the corresponding advantages of scientific research and talents. Therefore, it is necessary to strengthen the knowledge acquisition, attitude support and practical application of scientific research, to cultivate high level clinical and scientific research talents of rehabilitation of TCM, and promote the subject development of rehabilitation in TCM.
\end{abstract}

KEY WORDS TCM rehabilitation; scientific research level; knowledge-attitude-practice; investigation DOI: $10.3724 /$ SP.J.1329.2020.02013 